\title{
A Note on a Conjecture of L. J. Mordell
}

\author{
By Michael A. Malcolm*
}

Abstract. A computer proof is described for a previously unsolved problem concerning the inequality $\sum_{i=1}^{n} x_{i} /\left(x_{i+1}+x_{i+2}\right) \geqq n / 2$.

The function

$$
S_{n}(\mathbf{x})=\sum_{i=1}^{n} \frac{x_{i}}{x_{i+1}+x_{i+2}}, \quad(\text { subscripts } \bmod n)
$$

where $x_{i} \geqq 0, x_{i}+x_{i+1}>0, i=1,2, \cdots(\bmod n)$, has been studied by Shapiro, Mordell, and others (see [1]-[2], [6]-[11]). Let

$$
\lambda(n)=\inf _{\mathbf{x}} S_{n}(\mathbf{x})
$$

Then

$$
\lambda(n) \leqq n / 2 .
$$

H. S. Shapiro [10] suggested the verification of

$$
\lambda(n)=n / 2 .
$$

For $n \leqq 6$, several authors (see [6]) proved the validity of (4). Mordell [6] conjectured that (4) is false for all $n \geqq 7$, but later [7] stated that (4) is true for $n=7$. M. J. Lighthill (see [9]) and A. Zulauf [11] proved that (4) is false for $n=2 k, k \geqq 7$. D. Z. Djokovic [2] proved that (4) is true for $n=8$. P. H. Diananda [1] proved that (4) is false for $n=2 k+1, k \geqq 13$. In the same paper he proved (i) that if (4) is false for some $n=m$, where $m$ is odd, then (4) is false for all $n \geqq m$, and (ii) that if (4) is true for some $n=m$, where $m$ is even, then (4) is true for all $n \leqq m$. Recently, P. Nowosad [8] proved (4) is true for $n=10$, and thus (4) is true for all $n \leqq 10$.

Therefore, the question remains open for $n=12$ and for odd $n$ ranging from 11 to 25. In this note I will describe a computer proof that (4) is false for $n=25$.

The problem can be viewed as a multivariate constrained minimization of $S_{n}$. The constraints $x_{i} \geqq 0, i=1,2, \cdots, n$, can be removed by the simple change of variables

$$
x_{i}=\theta_{i}^{2}, \quad i=1,2, \cdots, n .
$$

If the second constraint is violated, i.e., $x_{i}+x_{i+1}=0$ for some $i$, the function (1) is undefined. Thus, the function

$$
S_{n}(\theta)=\sum_{i=1}^{n} \frac{\theta_{i}^{2}}{\theta_{i+1}^{2}+\theta_{i+2}^{2}},
$$

Received October 12, 1970.

AMS 1970 subject classifications. Primary 10-04, 10E20; Secondary 65K05, 65G05.

Key words and phrases. Cyclic sum, interval arithmetic.

* This work was sponsored in part by the Office of Naval Research (NR 044-211) and by the National Science Foundation (GJ 408). 
where the indices are taken mod $n$, can be minimized using a standard method. For the case $n=25$, this was done using a subroutine written by J. Alan George [3] on an IBM 360/67. The resulting values for $x_{i}$ were:

$$
\begin{array}{ll}
x_{1}^{*}=5.71138 & x_{13}^{*}=2.47011 \\
x_{2}^{*}=0 & x_{14}^{*}=3.15375 \\
x_{3}^{*}=6.76097 & x_{15}^{*}=1.66622 \\
x_{4}^{*}=1.10052 & x_{16}^{*}=3.058 \\
x_{5}^{*}=6.90241 & x_{17}^{*}=0.980738 \\
x_{8}^{*}=2.57379 & x_{18}^{*}=3.12582 \\
x_{7}^{*}=5.91561 & x_{19}^{*}=0.400648 \\
x_{8}^{*}=3.33613 & x_{20}^{*}=3.44328 \\
x_{9}^{*}=4.60951 & x_{21}^{*}=0 \\
x_{10}^{*}=3.47149 & x_{22}^{*}=4.07589 \\
x_{11}^{*}=3.43693 & x_{23}^{*}=0 \\
x_{12}^{*}=3.33360 & x_{24}^{*}=4.8248 \\
& x_{25}^{*}=0
\end{array}
$$

To prove that $S_{25}\left(\mathrm{x}^{*}\right)$ is less than 12.5 , the calculation (1) was programmed in a language [4] in which the calculations are carried out in interval arithmetic (see Moore [5]). The inaccuracies due to number conversion and roundoff are automatically accounted for by the language translator. Thus, assuming the translator is properly programmed, the resulting interval is guaranteed to contain the true result. The program was run on the IBM 360/67 at Stanford University, giving

$$
12.49847<S_{25}\left(x^{*}\right)<12.49851 \text {. }
$$

Computer time, including program compilations, amounted to 12 sec., costing exactly $\$ 1$. Programming time, including typing at a terminal, amounted to about four hours.

This method has been applied to the cases $n=23$ and $n=21$. In each case, an $\mathrm{x}$ could not be found such that $S_{n}(\mathrm{x})<n / 2$.

Computer Science Department

Stanford University

Stanford, California 94305

1. P. H. Diananda, "On a cyclic sum," Proc. Glasgow Math. Assoc., v. 6, 1963, pp. 1113. MR 27 \#87.

2. D. Z. Djokovic, "Sur une inégalité," Proc. Glasgow Math. Assoc., v. 6, 1963, pp. 110. MR 27 \#86.

3. J. AlAN George, FMIN1-Local Minimum of a Real Scalar Function of Several Variables Using a Gradient Search Method, Computer Science Department Program Library, Stanford University, Stanford, Calif., 1968. published.)

4. M. A. Malcolm \& J. Katzman, RAT-Range Arithmetic Translator, 1969. (Un- 
5. R. E. Moore, Interval Analysis, Prentice-Hall, Englewood Cliffs, N. J., 1966. MR 37 \#7069.

6. L. J. MORDELL, "On the inequality $\sum_{r=1}{ }^{n} x_{r} /\left(x_{r+1}+x_{r+2}\right) \geqq n / 2$ and some others," Abh. Math. Sem. Univ. Hamburg, v. 22, 1958, pp. 221-240.

7. L. J. MoRDELL, "Note on the inequality $\sum_{r=1}^{n} x_{r} /\left(x_{r+1}+x_{r+2}\right) \geqq n / 2$," J. London Math. Soc., v. 37, 1962, pp. 176-178. MR 25 \#2023.

8. PEDRo Nowosad, "Isoperimetric eigenvalue problems in algebras," Comm. Pure Appl. Math., v. 21, 1968, pp. 401-465. MR 38 \#6363.

9. R. A. RANKIN, "An inequality," Math. Gaz., v. 42, 1958, pp. 39-40.

10. H. S. Shapiro, "Problem 4603," Amer. Math. Monthly, v. 61, 1954, p. 571.

11. Von A. Zulauf, "Note on a conjecture of L. J. Mordell," Abh. Math. Sem. Univ. Hamburg, v. 22, 1968, pp. 240-241. 